separate needs. There is no Gender disaggregated data on IDUs available (UNODC 2004). Given that the socio-economic background and psychological conditions of FIDUs are different from their male counterparts (UNODC 2004), this study has been undertaken to study the sexual practises of and drug use pattern among FIDUs in Sikkim.

Methods The study has been conducted among FIDUs registered under the government projects operational in Sikkim. The sample had been selected on the basis of stratified random sampling. Structured Questionnaires had been developed and the Interviewers had been oriented on the research tool.

Results The study reveals that $73 \%$ of the FIDUs are sex workers, while $27 \%$ have multiple sexual partners. $36 \%$ inject drugs alone (70\% have suffered overdose) while $64 \%$ inject drugs in groups. $80 \%$ of the respondents consume both injecting and non-injecting drugs. $39 \%$ are daily drug users and the rest weekly. $38 \%$ and $42 \%$ are using drugs since for last 6 months and over one year respectively, highlighting that drug use is an age old practise and there are continuous new entries. Although only $10 \%$ of the respondents use condoms, $89 \%$ use oral contraceptives thereby reducing chances of pregnancy but risk of STI and HIV remain. $57 \%$ of the respondents have suffered from STI.

Conclusion Thus, the study reveals that convergence of drugs related services and sexual \& reproductive health services are essential. Unsafe injecting drug use combined with unprotected sex with multiple partners needs to be recognised and addressed through a comprehensive programme.

\section{P4.041 FACTORS ASSOCIATED WITH FREQUENT ALCOHOL DRINKING AMONG MEN WHO HAVE SEX WITH MEN: FINDINGS FROM A MULTI-SITE BIO-BEHAVIOURAL SURVEY IN INDIA}

doi:10.1136/sextrans-2013-051184.0939

'D Yadav, ${ }^{2}$ V Chakrapani, 'P Goswami, 'S Ramanathan, 'L Ramakrishnan, 'B George, 'S Sen, ${ }^{3} \mathrm{R}$ Paranjape. ${ }^{1} \mathrm{FHI}$ 360, New Delhi, India; ${ }^{2}$ Centre for Sexuality and Health Research and Policy (C-SHaRP), Chennai, India; ${ }^{3}$ National AIDS Research Institute (NARI), Pune, India

Background Men who have sex with men (MSM) in India are mostat-risk of HIV infection, with HIV prevalence almost 20 times higher than that in the general population. We describe the relationship between frequency of alcohol use and HIV-related risk behaviours among Indian MSM to develop strategies for addressing alcohol use within HIV interventions.

Methods Data for this analysis were drawn from a cross-sectional bio-behavioural survey (2009-2010) conducted in the states of Maharashtra, Tamil Nadu and Andhra Pradesh. 3,880 MSM were recruited from cruising sites using time-location cluster sampling. Binary logistic regression was carried out to assess relationships between frequent alcohol use and other sexual risk behaviours

Results We categorised those who reported consuming alcohol daily or at least once a week as 'frequent' drinkers ( $40 \%$ of the sample) and the remaining as 'infrequent' drinkers. Among frequent drinkers, majority were above 24 years (66\%), literate (85\%), self-identified as Kothis[feminine and receptive partner] (53\%), had paying male partners $(65 \%)$ and were exposed to HIV prevention interventions (78\%). Multivariate analysis showed that frequent alcohol drinkers were more likely to be above 24 years (adjusted odds ratio [AOR] 1.37, p < 0.05) and employed (AOR $1.54, \mathrm{p}<0.05)$. Frequent alcohol drinkers were less likely than infrequent drinkers to report consistent condom use with paying male partners (AOR 0.75, p < 0.05), consistent condom use with regular male partner (AOR 0.74, $p<0.05$ ), being community group member (AOR 0.84, p < 0.05), and self-identified as Kothi MSM (AOR 0.75, p < 0.05)
Conclusions Frequent alcohol drinking was more common among kothi-identified MSM and was significantly associated with inconsistent condom use with paying male partners and regular male partners. HIV prevention programmes need to be address alcohol consumption with better systematic screening counselling and referral to dependence treatment programme for men who have sex with men in India.

\section{P4.042 IGNORANCE! MAJOR CAUSE OF ADVANCED FORM OF GENITAL WARTS CLINICAL PRESENTATION IN KUMASI GHANA}

doi:10.1136/sextrans-2013-051184.0940

${ }^{1}$ T Agyarko-Poku, ${ }^{2} Y$ Adu Sarkodie, ${ }^{3} \mathrm{~L}$ Atakorah-Yeboah. 'Suntreso Government Hospital, Ghana Health Services, Kumasi, Ghana; ${ }^{2}$ School of Medical Sciences, Kwame Nkrumah University of Science and Technology, Kumasi, Ghana; ${ }^{3}$ Catholic University College, Fiapre, Sunyani, Ghana

Background Genital Wart is the most common clinical manifestation of genital Human papilloma virus infection. Early reporting of genital warts is the key to successful management. Presentation of more cases of an advanced form of genital warts to at Suntreso Sexually Transmitted Infection (STI) Clinic in Kumasi Ghana prompted this study to determine the possible reasons for the late reportage of genital warts conditions.

Methods 431 patients made up of $55.5 \%$ (239/431) females (F) and $44.5 \%(192 / 431)$ Males (M) who reported with an advanced form of genital warts- defined as warts affected more than one third of the external genital namely, vulva, vagina, peri-anal, penis and scrotum areas, with or without ulcerations- who consented to participate, were recruited for the cross sectional study. Participants were interviewed using structured questionnaire. Socoidemographic information was gathered. Data was analysed using SPSS 16.

Results $7.5 \%$ correctly mentioned that its genital warts (32/431; M- 9.9\%,19/192: F- 5.4\%). 46.1\% identified their condition as rashes (M-44.8\%, F- 46.4\%), $11.9 \%$ as said its cancer (M- 7.8\%, F-15.1), $14.6 \%$ as swelling (M-12.0\%,F- $16.7 \%$ ) while $19.9 \%$ had no idea about what is happening to their body (M-12.5\%, F- 25.5\%). Of the 42 respondents who have had tertiary education only $5(11.7 \%)$ could identify correctly genital warts.

Conclusion Very few people could identify genital warts showing a deficit in knowledge on genital warts among the population. The situation is not limited to only people with no formal education. Lack of knowledge on genital wart may be a precipitating factor in the delay reportage of cases. How patients perceived the causes of their conditions determines where and when to seek medical care in the era of medical plurarism. There is the need to step up education on genital warts among the population in Ghana.

\section{P4.043 THE MULTI-COMPONENT STUDY OF LOCATIONS, ESTIMATION SIZE AND BEHAVIOUR OF YOUNG HIGH RISK GROUPS IN ZAPORIZHZHIA CITY (UKRAINE) FOR DEVELOPMENT OF FOCUS INTERVENTION MODEL}

\section{doi:10.1136/sextrans-2013-051184.0941}

D Pavlova, Olga Balaireva, Tatiana Bondar, lana Sazonova, Olena Sakovych, Marissa Becker, Rob Lorway, S Isac. Ukrainian Institute for Social Research after Olexander Yaremenko, Kyiv, Ukraine

Background Ukraine's HIV prevalence is estimated at $1.6 \%$ of adult population. HIV epidemic in Zaporizhzhia is not extensive but tends to grow. The lack of knowledge about underage RG size is essential barrier for programme planning and service provision.

Methodology Team conducted multi-component research aimed at finding locations, size, typology and behaviour of risk groups aged 14-25. (i.e., FSWs, IDUs and street based children (SBC)). The 
research included geographic mapping of RG's locations, qualitative and quantitative surveys and mapping of existing services. Geographic mapping was based on interviews of primary $(\mathrm{N}=225)$ and secondary $(N=1240)$ key informants and showed more than thousand RGs locations. 125 representatives of each RG were interviewed during quantitative survey and 45 MARAs had in-depth interviews. Results Data shows that spots are very mobile which requires recurrent mapping. Street-based spots are popular locations for SBCs and FSWs. As a local peculiarity, mobile spots for IDUs were revealed, which means that drugs can be delivered by order. The preliminary data of size estimation in Zaporizhzhia shows that average number of FSW, IDUs and SBC is 2023, 2892 and 1388 relatively, what could be useful for planning and developing services. Obtained data shows high level of risk practises among MARAs (the percentage of condom use among FSWs with regular client is $65 \%$, with occasional clients - 76\%; the percentage of IDUs who have used only sterile syringes is $78 \%$ ). Range of HIV and Reproductive Health programmes revealed different types of services for MARAs with a poor access due to subjective reasons and geographical location of establishments.

Conclusions Research revealed the lack of HIV-prevention services for MARAs. For higher efficiency the developed HIV-prevention model should be very flexible. Service delivering process should be strongly supported by local government; the activity coordination and referral system should be thoroughly organised.

\section{P4.044 KNOWLEDGE AND ATTITUDES ABOUT HIV/AIDS AND SEXUAL HEALTH PRACTISES IN FIRST-YEAR UNIVERSITY STUDENTS}

doi:10.1136/sextrans-2013-051184.0942

\begin{abstract}
1,2B Sangchart, ${ }^{3,2}$ P Harnlakorn, ${ }^{4,2}$ P Kosalaraksa, ${ }^{5,2} \mathrm{C}$ Sota, ${ }^{6,2}$ S Barusrux, 7,2J Srijakkot, 8,2P Chetchotisak, 9,2S Chadbunchachai. 'Academic Nursing (Adult Nursing), Faculty of Nursing, Khon Kaen University, Khon Kaen, Thailand; '2AIDS Institute, Khon Kaen University, Khon Kaen, Thailand; ${ }^{3}$ Counseling Unit, Nursing Division, Srinagarind Hospital, Faculty of Medicine, Khon Kaen University, Khon Kaen, Thailand; "Department of Pediatrics, Faculty of Medicine, Khon Kaen University, Khon Kaen, Thailand; ${ }^{5}$ Department of Health Education, Faculty of Public Health, Khon Kaen University, Khon Kaen, Thailand; ' ${ }^{6}$ Department of Clinical Immunology and blood bank, Faculty of Associated Medical Sciences, Khon kaen University, Khon Kaen, Thailand; ${ }^{7}$ Academic Nursing (Nursing Administration), Faculty of Nursing, Khon Kaen University, Khon Kaen, Thailand; ${ }^{8}$ Department of Medicine, Faculty of Medicine, Khon Kaen University, Khon Kaen, Thailand; ' 9 Department of Social and Administrative Pharmacy, Faculty of Pharmaceutical Sciences, Khon Kaen University, Khon Kaen, Thailand
\end{abstract}

Background Good knowledge and attitudes about HIV/AIDS including safe-sex practises are important for adolescents' sexual health. The AIDS Institute at Khon Kaen University (KKU), promotes knowledge of HIV/AIDS and research into different strategies to reduce HIV/AIDS risk.

Method This study was conducted with first-year health-science KKU students. Sexual health behaviour, general knowledge about HIV and sexual transmitted diseases, HIV prevention beliefs, self-confidence and accessibility to care and counselling were explored using a self-report questionnaire, approved by KKU Ethics Committee.

Results Questionnaires were returned by 683 health-science students; $69.4 \%$ were female, mean age was 18.8 years. More than $90 \%$ of them declared that they have not had sexual experience. Many (74\%) had not talked about HIV with friends. Seven of ten survey questions about HIV knowledge were answered correctly in more than $84 \%$ of students. These questions included knowledge about at-risk populations, possibility of transmission without HIV symptoms, progression to death from opportunistic infection, transmission by eating together, timing for HIV testing, source of HIV in blood and body fluid and aggravated transmission by other sexual transmitted diseases. However, some still believed that HIV people should not have a sex life (33.7\%), or, did not know that coitus interruptus is unsafe for protecting from HIV infection (33.2\%). Regarding sexual practises, most were confident that they would not have sex without a condom (77.1\%), or, would be able to bargain not to have sex if they didn't want to $(82.5 \%)$, or, had access to condoms when needed (86.8\%)

Conclusion Students have good general knowledge about HIV/ AIDS. Most report confidence about only engaging in safe-sex behaviours, and having the communication skills to bargain with a partner to achieve this. However, behaviour in real life situations can be very different. This is difficult to research by self-report methods and would require other research tools.

\section{P4.045 WHEN TALKING OR NOT TALKING BECOMES A RISK: A GROUNDED THEORY STUDY EXPLORING THE IMPACT OF HIV ON IMMIGRANT BLACK AFRICAN FAMILIES IN THE UK}

doi:10.1136/sextrans-2013-051184.0943

J 0 A Owuor, A Locke, B Heyman. University of Huddersfield, Huddersfield, UK

Background Black Africans, culturally very diverse, make up less than $1 \%$ of the UK population. Yet they are the single most affected heterosexual group by HIV, accounting for a third of all annual new HIV diagnoses. Advancements in HIV treatment have transformed HIV into a chronic illness. Thus, families now deal with long term implications of HIV. In the UK, HIV testing, and more recently treatment, is free. Yet, black Africans predominantly test very late for HIV hence more susceptible to HIV-related morbidity and mortality. This paper explores the participants' risk perceptions, particularly men, on what it means to live with diagnosed HIV and the views of service providers on how the services can respond.

Methods This paper is based on a qualitative study involving indepth interviews with 23 participants; 11 positive men, 6 positive women, 1 negative man and 5 employees of HIV service-provider agencies. Data analysis was based on grounded theory's cyclic three-stage process; open coding, axial coding and selective coding.

Results The men and their partners rarely discussed their positive diagnosis with other family members, because they feared losing control over whom else would know about their diagnosis through a 'cascade of disclosure'. Although disclosure is a pre-requisite for seeking support, many participants shunned potential support rather than risk stigmatisation. Men were particularly reluctant to discuss their HIV status with other family members, and were more at risk of exposure. $83 \%$ of the men and $50 \%$ of the women had been exposed by a third party.

Conclusions Communication about HIV in the family, in a complex multicultural context, remains a challenge. This inhibits family members' access to and use of HIV services, contributing to continued poor outcomes for immigrant black Africans, particularly the men. This in turn diminishes the potential of the current positive prevention campaigns.

\section{P4.046 RISING PREGNANCY RATES AMIONG KNOWN HIV-POSITIVE WOMEN IN AT HEALTH CENTRES IN ADDIS ABABA, ETHIOPIA}

doi:10.1136/sextrans-2013-051184.0944

S Sisay, S Girma, T Arega, L Alamayehu, B Crandall, E L Konings. Management Sciences for Health, Addis Ababa, Ethiopia

Background At public health centres in Ethiopia, the proportion of known HIV-positive pregnant women at entry into ANC grew from $17 \%$ in 2009 to $36 \%$ in 2011 . To assess the reasons for this apparent rise in pregnancy among known HIV-positive women, we reviewed pregnancy rates among HIV-positive women of reproductive age (WRA) enrolled in HIV services. 